\title{
Natural infection of cattle with an atypical 'HoBi'-like pestivirus - Implications for BVD control and for the safety of biological products
}

\author{
Karl STÅHL ${ }^{\mathrm{a} *}$, Jaruwan KAMPA ${ }^{\mathrm{b}, \mathrm{c}}$, Stefan ALENIUS ${ }^{\mathrm{c}}$, Annie PERsSON \\ WADMAN $^{\mathrm{d}}$, Claudia BAULE ${ }^{\mathrm{a}}$, Suneerat AIUMLAMAI ${ }^{\mathrm{b}}$, Sándor BELÁK ${ }^{\mathrm{a}}$

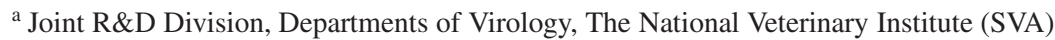 \\ \& The Swedish University of Agricultural Sciences (SLU), \\ Uppsala, Sweden \\ ${ }^{b}$ Faculty of Veterinary Medicine, Khon Kaen University, Thailand \\ ${ }^{c}$ Department of Clinical Sciences, The Swedish University of Agricultural Sciences (SLU), \\ Uppsala, Sweden \\ ${ }^{\mathrm{d}}$ Department of Virology, SVA, Uppsala, Sweden
}

(Received 6 October 2006; accepted 8 January 2007)

\begin{abstract}
During a study on Bovine Viral Diarrhoea (BVD) epidemiology in Thailand, a pestivirus was detected in serum from a calf. Comparative nucleotide sequence analysis showed that this virus was closely related to a recently described atypical pestivirus (D32/00_'HoBi') that was first isolated from a batch of foetal calf serum collected in Brazil. The results from virus neutralisation tests performed on sera collected from cattle in the herd of the infected calf, showed that these cattle had markedly higher antibody titres against the atypical pestivirus ' $\mathrm{HoBi}$ ' than against Bovine Viral Diarrhoea Virus types 1 and 2, or Border Disease Virus. The results also supported, consequently, the results from the molecular analysis, and demonstrated that a 'HoBi'-like pestivirus had been introduced to, and was now circulating in the herd. This study is the first to report a natural infection in cattle with a virus related to this atypical pestivirus, and it suggests that this group of pestiviruses may already be spread in cattle populations. The findings have implications for BVD control and for the biosafety of vaccines and other biological products produced with foetal calf serum. Consequently, these atypical pestiviruses should be included in serological assays, and any diagnostic assay aimed at detection of pestiviruses in biological products or animals should be tested for its ability to detect them.
\end{abstract}

BVDV / pestivirus / control / biosafety

\section{INTRODUCTION}

Bovine Viral Diarrhoea Virus (BVDV) is the denomination of a heterogeneous group of viruses in the family Flaviviridae, genus Pestivirus, which are eco-

* Corresponding author:

Karl.Stahl@bvf.slu.se, Karl.Stahl@sva.se nomically important pathogens worldwide, that primarily infect ruminants [11]. Currently there are two recognised genotypes of BVDV (BVDV-1, and -2) that together with Border Disease Virus (BDV) and Classical Swine Fever Virus (CSFV) constitute the four accepted species of the genus Pestivirus. In addition, a tentative fifth species is represented by 
a virus isolated from a giraffe [1]. The pestivirus genome consists of single stranded positive-sense RNA of approximately $12.3 \mathrm{~kb}$, with one open reading frame flanked by 5' and 3' non-coding regions (NCR). Classification of pestiviruses is based on the genetic relatedness between isolates, including genetic similarity with the type virus of the species [6]. Furthermore, because pestiviruses are serologically cross-reactive, species demarcation considers antigenic relationships based on binding assays with monoclonal antibodies or cross-neutralisation assays with polyclonal antisera $[1,5]$. Recently, an atypical pestivirus named D32/00_'HoBi' was isolated from foetal calf serum (FCS) originating from Brazil [13]. Based on genetic and antigenic properties that differed from previously described species within the genus, this virus was proposed as a representative of a possible novel pestivirus species. Furthermore, the results from in vitro and in vivo experiments suggested ruminants within the family Bovidae, genus $B o s$, as the most probable host species of this virus. This study is the first to report a natural infection in cattle with a virus related to this atypical pestivirus.

\section{MATERIALS AND METHODS}

\subsection{Virus origin}

A dairy herd was regularly checked serologically between 2000-2004 during a study on BVD epidemiology in Thailand [7]. All collected serum samples were tested for antibodies to BVDV at the Faculty of Veterinary Medicine and Animal Science, SLU, Sweden, using a commercial indirect ELISA (SVANOVA Biotech AB, Uppsala, Sweden). Seronegative samples were further analysed for BVDV antigen using a commercial antigen ELISA (Herd Check BVDV Ag/Serum Plus, IDEXX laboratories, Inc., Westbrook, ME,
USA). Due to Swedish legislation, all serum samples were heat inactivated before testing $\left(56{ }^{\circ} \mathrm{C}, 90 \mathrm{~min}\right)$. Between 2003 and 2004, 11 out of 16 previously seronegative animals seroconverted to BVDV without obvious clinical manifestations, indicating the introduction of a low virulent pestivirus into the herd. By using the antigen ELISA one seronegative viraemic calf was detected.

\subsection{Molecular methods}

For sequencing, RNA was extracted directly from the antigen positive serum using a GenoM-48 extraction robot (Geno Vision AS, Norway) and the extraction kit MagAttract Virus Mini M48 (Qiagen Gmbh, Germany) according to the standard protocol. A 296-nucleotide (nt) segment of the 5' NCR of the pestivirus genome was amplified in a one-step RT-PCR, as previously described [14], using the primers OPES 13A and OPES 14A [3]. The generated product was sequenced using the BigDye sequencing kit (BigDye ${ }^{\circledR}$ Terminator v3.1 Cycle Sequencing Kit, Applied Biosystems, Warrington, UK) according to the instructions of the manufacturer, using the same primers. A 183-nt fragment of the 5' NCR, corresponding to position 160-341 of BVDV NADL [2], was used for comparative sequence analyses. The nucleotide sequences were assembled and proofread using the SeqMan II and EditSeq programmes in the DNASTAR programme package (DNASTAR Inc., Madison, WI, USA), then aligned and compared by the Clustal W method of the MegAlign programme from the same package. Additional sequences representative of each known species within the genus Pestivirus, including the tentative pestivirus of giraffe, were obtained from the GenBank and included in the alignments. Phylogenetic trees were constructed 
using neighbour-joining [12], estimating evolutionary distances with the Kimura 2-parameter method [8]. The robustness of the method was evaluated by bootstrap resampling [4] with 1000 replicates. All phylogenetic analyses were performed using PAUP*, version 4.0 b10 [15].

\subsection{Virus neutralisation}

From the herd where the viraemic calf was detected, we selected serum samples (collected in 2004) from all animals that seroconverted between 2003 and 2004 ( $n=11)$, based on the results from the indirect ELISA. For comparison, we included randomly selected samples $(n=22)$ collected in 2001 from seropositive cattle in this and eight previously described herds in the region [7], and a serum sample (VD050916) used as a positive control for serological diagnosis within the Swedish BVD control programme, and that consists of pooled sera from a large number of BVDV-antibody positive Swedish cattle. The virus neutralisation (VN) capacity of all sera was tested against representatives from pestivirus species BVDV-1, BVDV-2, and D32/00_'HoBi', and those collected in 2004, together with the Swedish pooled serum, also against BDV. Two-fold dilutions of all sera were tested in duplicates from dilution 1:8. A fixed dose of virus $\left(100 \mathrm{TCID}_{50}\right)$ was mixed with $50 \mu \mathrm{L}$ sera and incubated in microtitre plates for $1 \mathrm{~h}$ at $37{ }^{\circ} \mathrm{C}$ with $5 \% \mathrm{CO}_{2}$. The cells $(50 \mu \mathrm{L}$ bovine turbinate cells, $3 \times 10^{5} / \mathrm{mL}$ ) were added and the cultures were incubated for 3 days at $37{ }^{\circ} \mathrm{C}$ with $5 \% \mathrm{CO}_{2}$. The plates were fixed and dried, then stained with immunoperoxidase as described by Meyling [10]. A VN antibody titre $\geqslant 8$ was considered a positive result, and the endpoint titre was determined by the dilution of serum that showed complete $\mathrm{VN}$ activity in both duplicates.

\section{RESULTS}

\subsection{Phylogenetic analysis}

The phylogenetic analysis clearly identified six major branches, supported by high bootstrap values, and demonstrated that the detected virus (named Th/04_KhonKaen ${ }^{1}$ ) was closely related to D32/00_'HoBi' (Fig. 1). The pairwise evolutionary distance within the studied fragment between Th/04_KhonKaen and D32/00_'HoBi' was estimated at 0.08. The ranges of pairwise distances between Th/04_KhonKaen and the other species within the genus Pestivirus, including the pestivirus of giraffe, were $0.28-0.43$ (Tab. I).

\subsection{Virus neutralisation}

All sera collected in 2004 had very high VN titres against D32/00_'HoBi'; in most cases markedly higher than against BVDV-1, BVDV-2 or BDV. Consequently, these results supported the results from the phylogenetic analysis, and clearly demonstrated that a 'HoBi'-like virus had been introduced to, and was now circulating in the herd. The Swedish serum and Thai sera collected in 2001, however, had high VN titres against NADL, but only low or moderate titres against the other pestiviruses (Tabs. II and III, respectively).

\section{DISCUSSION}

The heat-inactivation of all sera made virus isolation by conventional means impossible and, consequently, prevented further characterisation of Th/04_KhonKaen. However, sequence data and results from

\footnotetext{
${ }^{1}$ The GenBank/EMBL/DDBJ accession number for the 5' NCR sequence of isolate Th/04_KhonKaen reported in this paper is DQ897641.
} 


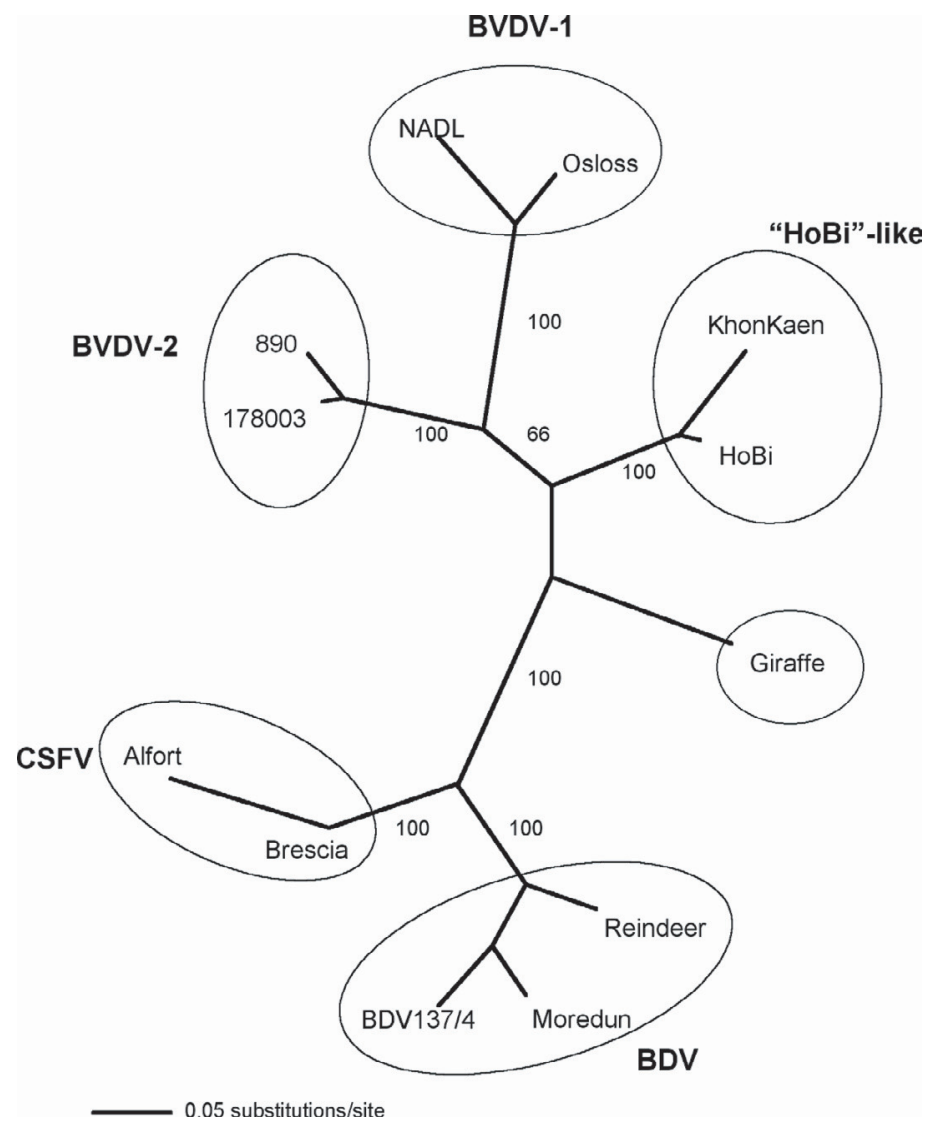

Figure 1. Unrooted phylogram generated from a 183-nucleotide fragment of the 5' NCR sequences of selected representatives of each known species within the genus Pestivirus, including the tentative pestivirus of giraffe, the D32/00_'HoBi' and Th/04_KhonKaen. The sequences of the virus strains NADL, Osloss, US890, Giraffe H138, Brescia, Alfort187, BDV137/4, Moredun, Reindeer-1 and D32/00_'HoBi' were obtained from the GenBank (accession nos. NC_001461, M96687, Z79772, AB040131, AF091661, X87939, U65052, U65022, NC_003677, AY489116). Strain CVL-178003/GB-1987 was kindly provided by T. Drew (VLA, UK) and included in the alignment. Values from bootstrap resampling with 1000 replicates are indicated for nodes with values above $60 \%$.

our serological studies clearly revealed the genetic and antigenic relationship between the virus that was circulating in the herd and D32/00_'HoBi', and supported the proposition of this group of viruses as representatives of a novel pestivirus species. The isolation of D32/00_'HoBi' from a batch of FCS from Brazil implied a risk of introduction of this atypical pestivirus into cattle populations through the use of biological products containing FCS [13]. The detection of a related virus in naturally infected cattle in Thailand suggests the following: (a) that this group of viruses has already been introduced to countries outside South America, possibly through 
Table I. Pairwise sequence distances based on a 183-nucleotide fragment of the 5' NCR sequences of selected representatives of each known species within the genus Pestivirus, including the tentative pestivirus of giraffe, the D32/00_'HoBi' and Th/04_KhonKaen.

\begin{tabular}{|c|c|c|c|c|c|c|c|c|c|c|c|c|}
\hline \multirow[t]{2}{*}{ Strain } & \multicolumn{12}{|c|}{ Pairwise sequence distance } \\
\hline & 1 & 2 & 3 & 4 & 5 & 6 & 7 & 8 & 9 & 10 & 11 & 12 \\
\hline 1. D32/00_HoBi & - & & & & & & & & & & & \\
\hline 2. Th/04_KhonKaen & 0.08 & - & & & & & & & & & & \\
\hline 3. BVDV-1 (NADL) & 0.35 & 0.36 & - & & & & & & & & & \\
\hline 4. BVDV-1 (Osloss) & 0.34 & 0.32 & 0.11 & - & & & & & & & & \\
\hline 5. BVDV-2 (890) & 0.25 & 0.31 & 0.27 & 0.28 & - & & & & & & & \\
\hline 6. BVDV-2 (178003) & 0.24 & 0.30 & 0.28 & 0.27 & 0.05 & - & & & & & & \\
\hline 7. BDV (Moredun) & 0.45 & 0.43 & 0.40 & 0.44 & 0.42 & 0.41 & - & & & & & \\
\hline 8. $\operatorname{BDV}(137 / 4)$ & 0.46 & 0.43 & 0.37 & 0.42 & 0.39 & 0.40 & 0.08 & - & & & & \\
\hline 9. Reindeer-1 & 0.39 & 0.38 & 0.34 & 0.38 & 0.36 & 0.38 & 0.12 & 0.11 & _- & & & \\
\hline 10. CSFV (Alfort187) & 0.40 & 0.43 & 0.46 & 0.46 & 0.49 & 0.48 & 0.33 & 0.39 & 0.32 & _- & & \\
\hline 11. CSFV (Brescia) & 0.37 & 0.37 & 0.34 & 0.33 & 0.41 & 0.39 & 0.24 & 0.29 & 0.22 & 0.11 & - & \\
\hline 12. Giraffe & 0.26 & 0.28 & 0.38 & 0.37 & 0.32 & 0.27 & 0.41 & 0.33 & 0.34 & 0.40 & 0.31 & - \\
\hline
\end{tabular}

Table II. Virus neutralisation titres of 11 sera (1-11) collected from cattle in a dairy herd in Thailand, 2004, and of one pooled serum (12) from Swedish cattle. Antibodies were detected against representative strains of pestivirus species BVDV-1, BVDV-2, BDV and the atypical pestivirus strain D32/00_'HoBi'.

\begin{tabular}{lcccc}
\hline Sera & \multicolumn{4}{c}{ Pestivirus strain } \\
\cline { 2 - 5 } & BVDV-1 $^{\mathrm{a}}$ & BVDV-2 $^{\mathrm{b}}$ & BDV $^{\mathrm{c}}$ & $\mathrm{HoBi}^{\mathrm{d}}$ \\
\hline 1 & 16 & 1024 & 128 & 8192 \\
2 & 32 & $<8$ & 64 & 8192 \\
3 & 64 & 128 & 16 & 2048 \\
4 & 64 & 512 & 8 & 8192 \\
5 & 64 & 2048 & 16 & 4096 \\
6 & 128 & 64 & 32 & 2048 \\
7 & 256 & 64 & 64 & 1024 \\
8 & 256 & 64 & 64 & 2048 \\
9 & 256 & 256 & 64 & 2048 \\
10 & 256 & 256 & 128 & 4096 \\
11 & 512 & 64 & 128 & 2048 \\
$12^{\mathrm{e}}$ & 1024 & 4 & 64 & 128 \\
\hline
\end{tabular}

a Strain NADL.

b Strain CVL-178003/GB-1987, kindly provided by

T. Drew, VLA, Addlestone, UK.

c Strain 137/4, kindly provided by T. Drew, VLA, Addlestone, UK.

d Kindly provided by M. Beer, Friedrich-LoefflerInstitut, Greifswald-Insel Reims, Germany.

e Pooled serum (VD050916) used as a positive control within the Swedish BVD control programme. contaminated vaccines produced with infected batches of FCS, or imported semen or embryos, and (b) that it may already be spread in cattle populations. The fact that pestiviruses are serologically cross-reactive and, at the same time, antigenically and genetically diverse [11], makes this suggestion feasible. It has been observed that, due to the genetic differences, the established pan-pestivirus PCR, using primer-pair 324/326 [16] might fail to detect 'HoBi'-like viruses [13]. Moreover, the serological cross-reactivity complicates differentiation through serological assays, as illustrated by the results of our VN studies. Had we not included D32/00_'HoBi' in the panel of viruses, the interpretation would have been that the herd was exposed to a mixed infection with BVDV-1 and -2 .

This first report of a natural infection in cattle with a 'HoBi'-like pestivirus indicates a risk that this atypical pestivirus, and others, may already be contaminants of live vaccines and other biological products, prepared for animal or human use. In addition, this finding has implications for any attempt to control BVD. In a country like Sweden, in the end phase 
Table III. Virus neutralisation titres of sera from 22 cattle, collected in 9 dairy herds in Thailand, 2001. Antibodies were detected against representative strains of pestivirus species BVDV-1, BVDV-2, and the atypical pestivirus strain D32/00_'HoBi'.

\begin{tabular}{lccc}
\hline Sera & \multicolumn{3}{c}{ Pestivirus strain } \\
\cline { 2 - 4 } & BVDV-1 $^{\text {a }}$ & BVDV-2 $^{\mathrm{b}}$ & HoBi $^{\mathrm{c}}$ \\
\hline 1 & $\geqslant 4096$ & 512 & 256 \\
2 & $\geqslant 4096$ & 512 & 256 \\
3 & $\geqslant 4096$ & 256 & 64 \\
4 & 512 & 64 & 32 \\
5 & 2048 & 32 & 16 \\
6 & 2048 & 128 & 256 \\
7 & $\geqslant 4096$ & 64 & 32 \\
8 & 512 & 512 & 16 \\
9 & 512 & 64 & 32 \\
10 & $\geqslant 4096$ & 64 & 8 \\
11 & $\geqslant 4096$ & 256 & 64 \\
12 & 1024 & 64 & 128 \\
13 & $\geqslant 4096$ & 128 & 512 \\
14 & 512 & 16 & 64 \\
15 & $\geqslant 4096$ & 1024 & 128 \\
16 & $\geqslant 4096$ & 64 & 128 \\
17 & 512 & $<8$ & $<8$ \\
18 & 1024 & 256 & 512 \\
19 & $\geqslant 4096$ & 512 & 512 \\
20 & $\geqslant 4096$ & 64 & 512 \\
21 & $\geqslant 4096$ & 8 & 256 \\
22 & $\geqslant 4096$ & 512 & 256 \\
\hline
\end{tabular}

a Strain NADL.

b Strain CVL-178003/GB-1987, kindly provided by T. Drew, VLA, Addlestone, UK.

${ }^{c}$ Kindly provided by M. Beer, Friedrich-LoefflerInstitut, Greifswald-Insel Reims, Germany.

of the BVD eradication programme [9], and with $>98 \%$ of the cattle population being free from and, thus, susceptible to BVDV, an introduction of atypical BVDV strains must be detected rapidly. If not, it would be a serious drawback that could have important economic consequences for the cattle industry. Therefore, we suggest that representatives of this group of viruses should be included in serological screening assays of cattle used in BVDV control programmes, and that live vaccines and other biological products should be tested for contamination with atypical pestiviruses to ensure good biosafety.

\section{REFERENCES}

[1] Becher P., Avalos Ramirez R., Orlich M., Cedillo Rosales S., König M., Schweizer M., Stalder H., Schirrmeier H., Thiel H.J., Genetic and antigenic characterization of novel pestivirus genotypes: implications for classification, Virology (2003) 311:96-104.

[2] Colett M.S., Larson R., Gold C., Strick D., Anderson D.K., Purchio A.F., Molecular cloning and nucleotide sequence of the pestivirus bovine viral diarrhea virus, Virology (1988) 165:191-199.

[3] Elvander M., Baule C., Persson M., Egyed L., Ballagi-Pordany A., Belak S., Alenius S., An experimental study of a concurrent primary infection with bovine respiratory syncytial virus (BRSV) and bovine viral diarrhoea virus (BVDV) in calves, Acta Vet. Scand. (1998) 39:251-264.

[4] Felsenstein J., Confidence limits on phylogenies: An approach using the boostrap, Evolution (1985) 39:783-791.

[5] Hamers C., Dehan P., Couvreur B., Letellier C., Kerkhofs P., Pastoret P.P., Diversity among bovine pestiviruses, Vet. J. (2001) 161:112-122.

[6] Heinz F.X., Collett M.S., Purcell R.H., Gould E.A., Howard C.R., Houghton M., Moormann R.J.M., Rice C.M., Thiel H.-J., Family Flaviviridae, in: van Regenmortel C.M.F.M.H.V., Bishop D.H.L., Carstens E.B., Estes M.K., Lemon S.M., Maniloff J., Mayo M.A., McGeoch D.J., Pringle C.R., Wickner R.B. (Eds.), Virus taxonomy: Seventh report of the International committee on taxonomy of viruses, Academic Press, San Diego, CA, 2000.

[7] Kampa J., Ståhl K., Moreno-Lopez J., Chanlun A., Aiumlamai S., Alenius S., BVDV and BHV-1 infections in dairy herds in northern and northeastern Thailand, Acta Vet. Scand. (2004) 45:181-192.

[8] Kimura M., A simple method for estimating evolutionary rates of base substitutions through comparative studies of nucleotide sequences, J. Mol. Evol. (1980) 16:111-120. 
[9] Lindberg A.L., Alenius S., Principles for eradication of bovine viral diarrhoea virus (BVDV) infections in cattle populations, Vet. Microbiol. (1999) 64:197-222.

[10] Meyling A., Detection of BVD virus in viremic cattle by an indirect immunoperoxidase technique, in: McNulty M.S., MacFerran J.B. (Eds.), Recent advances in virus diagnosis, Martinus Nijhoff, Boston, 1984.

[11] Ridpath J.F., BVDV genotypes and biotypes: practical implications for diagnosis and control, Biologicals (2003) 31:127-131.

[12] Saitou N., Nei M., The neighbor-joining method: a new method for reconstructing phylogenetic trees, Mol. Biol. Evol. (1987) 4:406-425.

[13] Schirrmeier H., Strebelow G., Depner K., Hoffmann B., Beer M., Genetic and antigenic characterization of an atypical pestivirus isolate, a putative member of a novel pestivirus species, J. Gen. Virol. (2004) 85:3647-3652.

[14] Ståhl K., Kampa J., Baule C., Isaksson M., Moreno-Lopez J., Belak S., Alenius S., Lindberg A., Molecular epidemiology of bovine viral diarrhoea during the final phase of the Swedish BVDeradication programme, Prev. Vet. Med. (2005) 72:103-108; discussion 215-219.

[15] Swofford D.L., PAUP*. Phylogenetic analysis using parsimony (* and other methods), Version 4, Sinauer Associates, Sunderland, Massachusetts, 2003.

[16] Vilcek S., Herring A.J., Herring J.A., Nettleton P.F., Lowings J.P., Paton D.J., Pestiviruses isolated from pigs, cattle and sheep can be allocated into at least three genogroups using polymerase chain reaction and restriction endonuclease analysis, Arch. Virol. (1994) 136:309-323. 